\title{
Manejo de epistaxis posterior en el Hospital Clínico de la Universidad de Chile entre los años 2013 y 2016
}

\section{Treatment of posterior epistaxis in the Clinical Hospital of the University of Chile between the years 2013 and 2016}

\author{
Daniel Retuert R', David Fuentealba $\mathbf{D}^{1}$, Andrea Bretón $\mathrm{I}^{2}$, Lara Ricci $\mathbf{L}^{3}$, Rodolfo Nazar $\mathbf{S}^{1}$, \\ Alfredo Naser G'.
}

\begin{abstract}
RESUMEN
Introducción: La epistaxis corresponde a una de las causas más frecuentes de consulta otorrinolaringológica en los servicios de urgencia. La epistaxis posterior es menos frecuente pero su presentación más severa. Existen varias alternativas terapéuticas, en las últimas décadas el manejo quirúrgico endoscópico de la arteria esfenopalatina (AEP) ha ido en aumento dado las ventajas en comodidad para el paciente y reducción de costos asociados.

Objetivo: Revisar el manejo realizado en los cuadros de epistaxis posterior en el Hospital Clínico de la Universidad de Chile (HCUCh).

Material y método: Estudio descriptivo en el Servicio de Otorrinolaringología del HCUCh de pacientes que presentaron epistaxis posterior entre el año 2013 y 2016.

Resultados: Se revisó un total de 33 casos. La edad promedio de los pacientes fue 61,6 años siendo las comorbilidades más frecuentes la hipertensión arterial $(36,3 \%)$ y fibrilación auricular (18,1\%). Se realizó tratamiento quirúrgico en $57,6 \%$ de los pacientes siendo el clipaje de AEP la intervención quirúrgica más frecuente.

Discusión y conclusiones: Tanto el manejo tradicional como las técnicas quirúrgicas presentaron eficacia similar en la serie revisada.

Palabras clave: Epistaxis, epistaxis posterior, ligadura endoscópica arteria esfenopalatina, cirugía endoscópica nasal.
\end{abstract}

\section{ABSTRACT}

Introduction: Epistaxis corresponds to one of the most frequent causes of otorhinolaryngological consultation in the emergency services. Posterior epistaxis is less frequent but more severe. There are several therapeutic alternatives, in recent decades the surgical endoscopic management of the sphenopalatine artery (AEP) has been increasing given the advantages in comfort for the patient and reduction of associated costs.

\footnotetext{
${ }^{1}$ Médico Servicio de Otorrinolaringología Hospital Clínico Universidad de Chile.

${ }^{2}$ Médico Cirujano en Etapa de Destinación y Formación Hospital Achao, Servicio de Salud Chiloé.

${ }^{3}$ Interna de Medicina de séptimo año, Universidad de Chile.
} 
Aim: To review the management of posterior epistaxis in the Clinical Hospital of the University of Chile (HCUCh).

Material and method: Descriptive study in the Otorhinolaryngology Service of the HCUCh of patients who presented posterior epistaxis between the years 2013 and 2016.

Results: $A$ total of 33 cases were reviewed. The mean age of the patients was 61.6 years, with the most frequent comorbidities being hypertension (36.3\%) and atrial fibriIlation (18.1\%). Surgical treatment was performed in $57.6 \%$ of the patients, with AEP clipping being the most frequent surgical intervention.

Conclusion: Both traditional management and surgical techniques presented similar efficacy in the revised series.

Key words: Epistaxis, posterior epistaxis, endoscopic ligation, sphenopalatine, nasal endoscopic surgery.

\section{INTRODUCCION}

La epistaxis, definida como episodio de sangrado activo por la nariz, corresponde a una de las causas más frecuentes de admisión de urgencia al servicio de otorrinolaringología ${ }^{1}$. Corresponde a una patología frecuente, el $60 \%$ de la población sufre algún episodio de epistaxis en su vida pero tan sólo $6 \%$ requiere atención médica para tratamiento y control hemostático ${ }^{3}$. De este grupo, sólo 1,6 por cada 100.000 pacientes serán hospitalizados para el manejo, ocurriendo en su mayoría en pacientes entre 60 y 70 años. La incidencia de epistaxis en la población tiene un comportamiento bimodal con una presentación frecuente en las edades pediátricas menores de 10 años y luego un alza en adultos a partir de los 35 años ${ }^{1,2}$.

Se estima que cerca del $90 \%$ de las epistaxis ocurren en el septo nasal anterior 0 area de Kiesselbach y sólo entre $5 \%$ y $10 \%$ son posteriores con origen en el septo nasal 0 en la pared lateral, cuya irrigación principal proviene de la arteria esfenopalatina y sus ramas. Esta última causa es más frecuente en pacientes adultos mayores con un promedio de edad de 64 años en estudios retrospectivos ${ }^{1,3}$. Por la severidad de la epistaxis posterior, su manejo significa un gran desafío, existiendo varias alternativas de tratamiento. En las últimas décadas el manejo quirúrgico como cauterización o ligadura endoscópica de la arteria esfenopalatina (AEP) ha ido en incremento ya que al comparar con el taponamiento, el manejo de la AEP disminuye los tiempos de estadía hospitalaria, mejora la satisfacción del paciente y reduce los $\operatorname{costos}^{1,4}$.
A pesar de la frecuencia de esta patología, su enfrentamiento varía entre distintos centros ya que no existen prácticas estandarizadas en forma global ${ }^{1}$. Este estudio analiza el manejo de las epistaxis posteriores del Servicio de Otorrinolaringología del HCUCh entre los años 2013-2016.

\section{OBJETIVOS}

Describir el manejo realizado en los cuadros de epistaxis posterior de pacientes hospitalizados en el HCUCH y evaluados por el Servicio de Otorrinolaringología entre los años 2013 y 2016, junto con el análisis de variables tales como edad, sexo, causas predisponentes, comorbilidades y tiempos de estadía hospitalaria, con la finalidad de desarrollar y perfeccionar protocolos de manejo de epistaxis posterior en pacientes hospitalizados en el HCUCH.

\section{MATERIAL Y METODO}

Estudio retrospectivo, descriptivo y transversal. Pacientes egresados del HCUCH con diagnóstico de epistaxis posterior entre los años 2013 y 2016 y que fueron evaluados por el Servicio de Otorrinolaringología $\mathrm{HCUCH}$, siendo estos pacientes propios del Servicio de Otorrinolaringología $\mathrm{HCUCH}$ o de otro servicio del HCUCH. Para ello se revisaron fichas clínicas electrónicas y protocolos operatorios. En el análisis se consideraron variables epidemiológicas como género, edad, comorbilidades, tratamiento realizado, estadía 
hospitalaria y complicaciones asociadas al procedimiento o durante el posoperatorio.

El análisis de datos se obtuvo mediante programa Excel obteniendo variables cuantitativas descritas mediante promedios y sus respectivas desviaciones estándar.

Se realizó una búsqueda bibliográfica en bases de datos electrónicas a través de Pubmed y Epistemonikos con los términos epistaxis, epistaxis posterior, arteria esfenopalatina, cirugía endoscópica nasal.

\section{RESULTADOS}

Se revisó un total de 33 casos de pacientes egresados del HCUCH con diagnóstico de epistaxis posterior entre los años 2013 y 2016 y que fueron evaluados por el Servicio de Otorrinolaringología HCUCH. La edad promedio de los pacientes incluidos en el estudio fue 61,64 años (DS 19,94), correspondiendo a 32 adultos y sólo 1 caso pediátrico de 6 años. El rango etáreo de los pacientes adultos fue entre 19 y 97 años. De los casos ingresados 54,6\% correspondieron a pacientes de sexo masculino y $45,4 \%$ de sexo femenino. Las comorbilidades más frecuentes fueron hipertensión arterial (HTA) en $36,3 \%$ y fibrilación auricular (FA) en $18,1 \%$. De los 6 pacientes que se presentaron con FA, 5 se encontraban en terapia anticoagulante oral (TACO) al momento de presentación de epistaxis y 1 caso con antecedente de suspensión de TACO 20 días previo al episodio de sangrado. Otras comorbilidades relevantes encontradas en esta serie fueron diabetes mellitus 2 (DM2), cardiopatía coronaria, tromboembolismo pulmonar (TEP) y lupus eritematoso sistémico (LES) (Figura 1).

En $62,7 \%$ de los casos se identificaron factores de riesgo, presentándose en orden de frecuencia: uso de aspirina, posquirúrgico, TACO, trombocitopenia, desviación septal, fractura nasal, cirugía endoscópica funcional asociada a anticoagulación (Figura 2). En el $27,2 \%$ restante de los casos no se logró identificar factores predisponentes.

Respecto al manejo definitivo, el $42,4 \%$ de los casos fueron manejados sólo con taponamiento posterior y $57,6 \%$ requirió intervención quirúrgica. De los pacientes operados, en $84 \%$ se realizó manejo de la AEP mientras que el $15 \%$ requirió otro tipo de intervención quirúrgica como ligadura de arteria septal posterior, septoplastía más antrostomía del seno maxilar izquierdo y adenoidectomía en el paciente pediátrico.

El promedio de estadía hospitalaria en nuestra serie fue de 5,3 días, siendo necesario mencionar un número importante de pacientes con patología cardiovascular asociada que requirieron manejo de sus patologías de base, 15,1\% de pacientes en TACO y el caso de una paciente con LES presentó hospitalización prolongada de 30 días por el manejo de complicaciones de su patología de base, no relacionada con epistaxis. El promedio de estadía hospitalaria de los pacientes que requirieron intervención quirúrgica fue 4,1 días y en los no operados 6,9.

La mayoría de los pacientes evolucionaron favorablemente, sólo dos (6\%) presentaron resan-

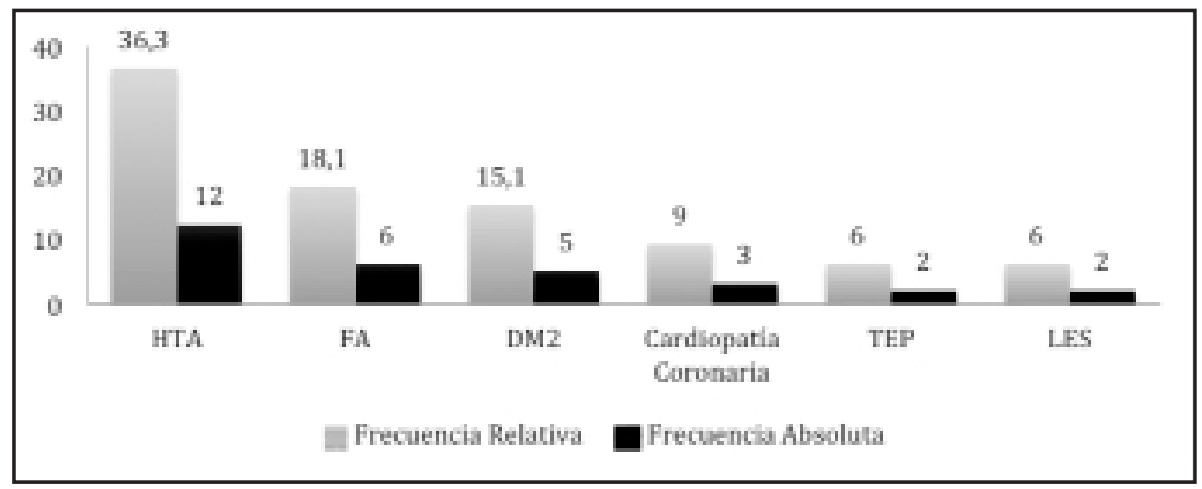

Figura 1. Comorbilidades en pacientes con epistaxis posterior. 


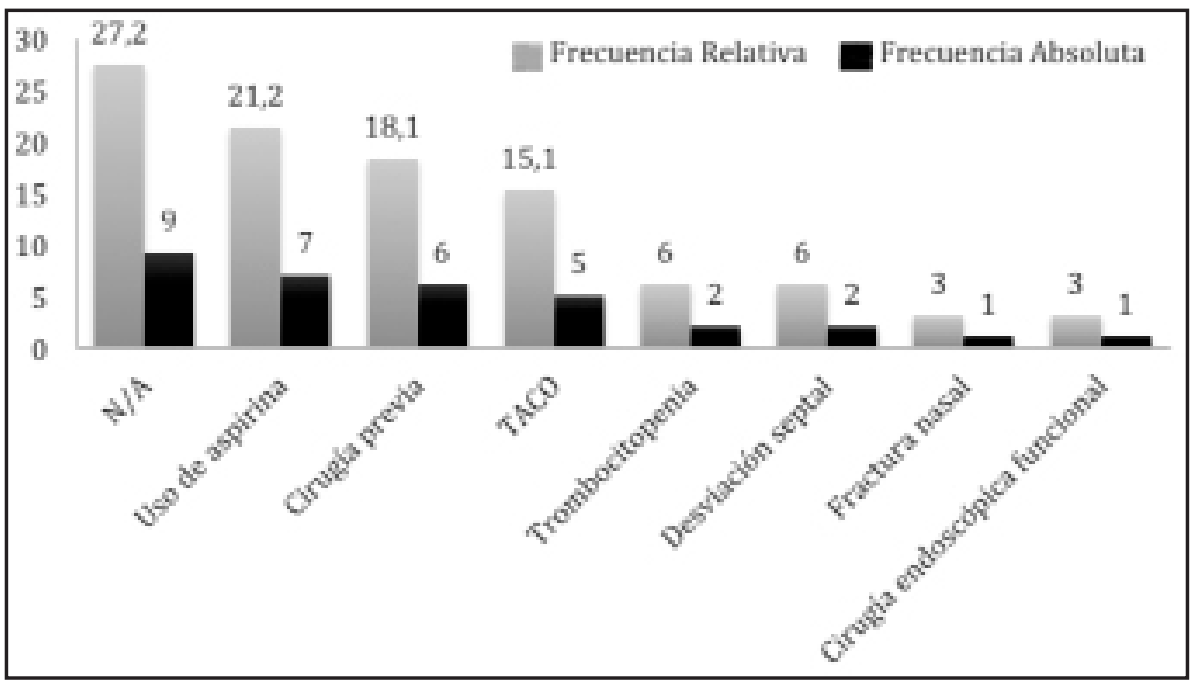

Figura 2. Factores de riesgo de epistaxis posterior.

grado, ambos posterior a ligadura AEP, requiriendo en un caso cauterización de arteria septal con nitrato de plata y el otro nueva ligadura de AEP. Un caso presentó escara del ala nasal secundaria a taponamiento. Dos pacientes fallecieron, ambos por causas no relacionadas con la epistaxis (leucemia y miocardiopatía hipertrófica).

\section{DISCUSION}

Concordante con lo descrito en la literatura, la epistaxis posterior fue más frecuente en pacientes adultos $(96,9 \%)$, no encontrándose diferencia significativa por género. La principal causa predisponente fue uso de aspirina, seguida por causa posquirúrgica y uso de TACO. En nuestro estudio, el manejo definitivo fue quirúrgico en el $57,5 \%$ de los casos, siendo el abordaje de AEP la cirugía más frecuente $(84,2 \%)$.

Dado un número importante de pacientes hospitalizados con patología cardiovascular asociada, el taponamiento posterior como terapia definitiva se realizó en 14 pacientes (42,4\%).

Si bien nuestro estudio muestra una eficacia similar entre el manejo médico y quirúrgico en el control de la epistaxis, han sido descritas en numerosos estudios las ventajas de esta última intervención ${ }^{4-7}$.
El manejo endoscópico nasal permite localizar el sitio de sangrado, cuando esto es posible se realiza cauterización del sitio de sangrado que no requiere taponamiento posterior. Sin embargo, esta técnica tiene menor tasa de efectividad que la ligadura de AEP probablemente por identificación inadecuada del sitio de sangrado 5 . En la ligadura, la eficacia depende del control de las ramas de la AEP, por lo que la indicación de ligadura de la AEP incluye la imposibilidad de taponamiento efectivo por deformidad anatómica, falla en terapia no quirúrgica, epistaxis recurrente.

Actualmente la indicación de cirugía endoscópica nasal se ha considerado como alternativa al taponamiento dado que al comparar con técnicas tradicionales no quirúrgicas, la ligadura de AEP ha demostrado mejor satisfacción del paciente, reducción de la estadía hospitalaria y los costos asociados $^{1,4}$. En 2 revisiones sistemáticas de 2003 y $2005^{8,9}$ que evaluaron la efectividad de la ligadura de AEP en 127 y 264 pacientes respectivamente, se obtuvieron resultados de $98 \%$ y $70 \%-100 \%$ de efectividad. Las complicaciones descritas de la ligadura AEP son costras nasales, disminución del lagrimeo, parestesia del paladar 0 de la nariz, perforación septal y necrosis de cornetes inferiores ${ }^{1}$.

En la actualidad, una alternativa para casos de epistaxis posterior que no cede con la ligadura 0 cauterización arterial quirúrgica, es la embolización 
AEP o sus ramas. Sin embargo, esta técnica está reservada para casos refractarios dado el potencial riesgo de accidente cerebrovascular, disección arterial, isquemia facial, entre otros descritos ${ }^{1,10}$. Un estudio recientemente publicado ${ }^{11}$ evidenció que la ligadura en comparación con embolización arterial disminuye los costos y acorta la hospitalización sin incrementar las tasas de complicaciones.

Frente a la diversidad de terapias disponibles para el manejo de epistaxis posterior, se ha propuesto el uso de protocolos estandarizados que permitan mejorar el manejo de los pacientes con respecto a la costo-efectividad de los tratamientos. El estudio prospectivo de Vosler y cols ${ }^{12}$ comparó el manejo de epistaxis severa (no controlable con compresión, vasoconstrictores locales o taponamiento anterior) antes y después de la aplicación de un protocolo. Este estudio mostró, posterior a la implementación del protocolo, un aumento de ligaduras de la AEP, disminución del número de días de taponamiento, disminución estadía hospitalaria (de 5,2 a 2,1 días) y disminución del costo total

\section{BIBLIOGRAFIA}

1. M.L. Barnes, P.M. Splelmann, P.S. White. Epistaxis: A Contemporary Evidence Based Approach. Otolaryngol Clin 2012; 45: 1005-17.

2. Purkey MR, Seeskin Z, Chandra R. Seasonal variation and predictors of epistaxis. Laryngoscope 2014; 124(9): 2028-33.

3. Rodney J. SCHLosser, M.D.N Epistaxis. Engl J Med 2009; 360: 784-9.

4. Moshaver A, Harris JR, Liu R, et al. Early operative intervention versus conventional treatment in epistaxis: randomized prospective trial. $J$ Otolaryngol 2004; 33: 185-8.

5. McClurg SW, Carrau R. Endoscopic management of posterior epistaxis: a review. Acta Otorhinolaryngologica Italica 2014; 34(1): 1-8.

6. Paul J, Kanotra SP, Kanotra S. Endoscopic management of posterior epistaxis. Indian $J$ Otolaryngol Head Neck Surg 2011; 63: 141-4.

7. Dedhia RC, Desal SS, Smith KJ, et al. Costeffectiveness of endoscopic sphenopalatine artery ligation vs. nasal packing as first-line de hospitalización. Posterior al inicio del protocolo ningún paciente recibió embolización.

\section{CONCLUSION}

Tanto el manejo endoscópico mediante ligadura o cauterización de AEP como el taponamiento posterior son eficaces en el control de epistaxis posterior. En nuestro centro, ambos tipos de tratamiento han sido utilizados exitosamente en el manejo de pacientes hospitalizados que presentan epistaxis posterior. La elección del tratamiento sigue siendo una decisión individualizada en la que se deben considerar variables como comorbilidades, tratamientos concomitantes y causa más probable de sangrado. En los nuevos protocolos propuestos para el manejo de epistaxis, se consideran cada vez más las técnicas quirúrgicas en forma precoz, dado sus ventajas al mejorar la satisfacción del paciente, reducir los días de hospitalización y los costos asociados.

treatment for posterior epistaxis. Int Forum Allergy Rhinol 2013; 3: 563-6.

8. Kumar S, Shetty A, Rockey J, Nilssen E. Contemporary surgical treatment of epistaxis. What is the evidence for sphenopalatine artery ligation? Clinical Otolaryngology and Allied Sciences 2003; 28(4): 360-3.

9. Feusi B, Holzmann D, Steurer J. Posterior epistaxis: systematic review on the effectiveness of surgical therapies. Rhinology 2005; 43(4): 300-4.

10. BrinjikJ W, Kallmes DF, Cloft HJ. Trends in epistaxis embolization in the United States: a study of the Nationwide Inpatient Sample 2003-2010. J Vasc Interv Radiol 2013; 24(7): 969-73.

11. Sylvester M.J, Chung S.Y, Guinand, L.A, Govindan A, Baredes S, Eloy J.A. Arterial ligation versus embolization in epistaxis management: Counterintuitive national trends. The Laryngoscope 2017; 127: 1017-20.

12. Peter S. Vosler et al. Successful Implementation of a Clinical Care Pathway for Management of 
Epistaxis at a Tertiary Care Center. Otolaryngol Head Neck Surg 2016; 155(5): 879-85.

13. Christensen NP, Smith DS, Barnwell SL, et al. Arterial embolization in the management of posterior epistaxis. Otolaryngol Head Neck Surg 2005; 133: 748-53.

14. Soyka MB, Nikolaou G, Rufibach K, et al. On the effectiveness of treatment options in epistaxis: an analysis of 678 interventions. Rhinology 2011; 49: 474-8.

15. Rezende GL, Soares Vy, Moraes WC, et al. The sphenopalatine artery: a surgical challenge in epistaxis. Braz J Otorhinolaryngol 2012; 78: 42-7.

16. Nikolaou G, Holzmann D, Soyka MB. Discomfort and costs in epistaxis treatment. Eur Arch Otorhinolaryngol 2013; 270: 2239-44.

17. Douglas R, Wormald PJ. Update on epistaxis. Curr Opin Otolaryngol Head Neck Surg 2007; 15: 180-3.

18. Kumar S, Shetty A, Rocker J, et al. Contemporary surgical treatment of epistaxis. What is the evidence for SPA ligation? Clin Otolaryngol Allied Sci 2003; 28: 360-3.

Dirección : Daniel Retuert $\mathrm{R}$

Hospital Clínico Universidad de Chile

E mail: danielretuert@gmail.com 